\title{
Comparison of stepping parameters and center of foot pressure properties during different tempo stepping movements
}

\author{
Shunsuke Yamaji ${ }^{\star}$, Shinichi Demura ${ }^{2}$, Sohee Shin $^{3}$, Hiroki Aoki ${ }^{4}$, Yamamoto Yuta ${ }^{5}$ \\ ${ }^{1}$ Faculty of Medical Sciences, University of Fukui, Fukui, Japan; *Corresponding Author: yamaji@fmsrsa.fukui-med.ac.jp \\ ${ }^{2}$ Graduate School of Natural Science \& Technology, Kanazawa University, Kanazawa, Japan \\ ${ }^{3}$ School of Medicine, Gifu University, Gifu, Japan \\ ${ }^{4}$ Kanazawa College of Art, Kanazawa, Japan \\ ${ }^{5}$ Kanazawa Gakuin High School, Kanazawa, Japan
}

Received 10 July 2012; revised 8 August 2012; accepted 18 August 2012

\begin{abstract}
This study was designed to examine the trialto-trial reliability of stepping parameters and to compare the center of foot pressure (COP) when adapting to different tempos. Twenty healthy men ( $\mathrm{M}$ age $=20.8, \mathrm{SD}=1.9$ years) performed stepping tests to fixed tempos. The intra-class correlation coefficients of stepping and COP parameters were high $(0.75-0.97)$. The time difference between foot contact time and fixed tempo and COP sway velocities of the $\mathrm{X}$ - and $\mathrm{Y}$-axis were the largest at 40 beats per minute (bpm). At 60 and $120 \mathrm{bpm}$ tempos, single support and swing times reflecting the stepping strategy correlated significantly with the root mean square and the velocity of the $X$-axis $(r=0.45-0.53 \mid)$. Because these step tempos are controlled largely by the automation of stepping, the single support and swing phases appeared to have a constant frequency. On the other hand, a stepping tempo of $\mathbf{4 0} \mathrm{bpm}$ showed the largest sway velocity of $X$ and $Y$ and the smallest surrounding area mean path length. The above suggests that stepping at $\mathbf{4 0} \mathrm{bpm}$ induced a larger sway area in relation to total path length and faster COP sway. Hence, it is inferred that the stepping task becomes more difficult at $40 \mathrm{bpm}$ than at 60 and $120 \mathrm{bpm}$.
\end{abstract}

Keywords: Dynamic Balance; Trial-to-Trial Reliability; Fall Prevention

\section{INTRODUCTION}

Improving the independence of the elderly is an urgent issue in many countries due to the increasing aging rate of the population. Prevention of bone fractures caused by falls is important as well [1,2]. Falls are the result of a complex set of factors including physical function, environment, disease, and medications [3]. Because the improvement of physical function with appropriate exercise is very effective for fall prevention in healthy elderly people, most fall prevention programs aim mainly to improve physical function [1,4]. In particular, walking is included in most programs because independent dailyliving and fall risk depend largely on mobility.

Mobility tests have been proposed in many previous studies (10 m walk test [5], Tandem gait test [6], Figure eight track walk test [7], Timed up \& Go test [8], Obstacle walk test [9], Maximal stepping test [10-12]. These tests are useful for the evaluation of daily-living activeties of the elderly. However, falls occur when a stable body posture cannot be kept after an accidental disturbance [1]. It is difficult to simulate the accidental disturbances that induce a fall in the above stated general mobility tests because of their predictable walking environment $[12,13]$. In addition, it will be important to evaluate the capacity to control for accidental disturbances during ambulation.

The control of body sway based on disturbances during movement depends on the postural control system consisting of the labyrinth, visual, and somatosensory systems in addition to lower limb strength to support the swaying body. The ability to maintain the body's center of gravity within a supportable area and maintain stability during a movement despite accidental disturbances is defined as dynamic balance [12,13]. Previous studies have examined dynamic balance ability during physical disturbances using floor tilt, floor vibration, or a traction apparatus [14], but these are not practical or safe for use as a general exercise or evaluation test. 
To improve safety, Demura et al. [15] and Shin and Demura [16] proposed stepping tests with fixed tempo in which subjects adapt to tempos slower than the typical walking tempo. The above tasks require extension of the one leg support phase. A person with inferior dynamic balance ability will find it difficult to maintain stable body posture during this phase. The ability to adapt to fixed tempos depends largely on the tempo. Shin and Demura [16] compared stepping properties at 40,60 , and 120 beats per minute (bpm) tempos for the young and the elderly and clarified that the time difference between the stepping movement and tempo is the largest at the 40 bpm tempo in both groups. Moreover, they reported that the stepping strategy, in short, the ratio of one leg support and both leg support phases, differs with tempo. The extension of the one leg support phase at slow tempos is assumed to make maintenance of body balance more difficult. On the other hand, stepping at fast tempos is considered to induce large amounts of body sway, although the effect of tempo on body sway has not been examined.

This study aimed to clarify the trial-to-trial reliability of stepping parameters and to compare the center of foot pressure (COP) when adapting to different tempos (40, 60, and $120 \mathrm{bpm})$.

\section{METHOD}

\subsection{Participants}

Twenty healthy males aged 18 - 25 yr participated in this study. Table 1 shows characteristics of participants in this study. Informed consent was obtained from each subject after a full explanation of the experimental project and its procedure. This study was approved by the Kanazawa University Ethics Committee.

\subsection{Materials}

A gait analysis meter (Walkway MG-1000, Anima and Japan) was used for the step test. This device can measure in real time when the subject's right or left foot touches and takes off from the step sheet based on foot pressure information. The sampling frequency was 100 $\mathrm{Hz}$. The subjects stood on the step sheet and stepped to match the tempo of a metronome. A $120 \mathrm{bpm}$ tempo was reported to be the most efficient interval during walking [17]. Sixty bpm and 40 bpm tempos, which correspond to $1 / 2$ and $1 / 3$ intervals of $120 \mathrm{bpm}$, were selected as slower tempos [16].

Table 1. Characteristics of participants $(n=20)$ in this study.

\begin{tabular}{cccc}
\hline & & Mean & SD \\
\cline { 3 - 4 } Age & (years) & 20.8 & 1.9 \\
Height & $(\mathrm{cm})$ & 170.6 & 3.7 \\
Body mass & $(\mathrm{kg})$ & 65.3 & 4.7 \\
\hline
\end{tabular}

An Anima's stabilimeter (G5500, Japan) was used for the measurement of COP. This instrument can calculate the COP of vertical loads from values of three vertical load sensors that are put on the peak of an isosceles triangle on a level surface. This instrument was placed under the gait analysis meter. Data measured in both systems were recorded at a sampling frequency of $20 \mathrm{~Hz}$.

\section{Experimental Procedure}

To eliminate order effect, both tests were randomly allotted to each subject. After one practice run, they performed each test for $20 \mathrm{~s}$ twice with a 1 min rest. The subjects stood on the step sheet and stepped with their left or right foot alternately while adapting to the abovestated tempos.

\subsection{COP Parameters}

Five parameters for COP sway were selected to evaluate body sway during stepping as follows: root mean square of $\mathrm{X}$ and $\mathrm{Y}$-axis, area surrounding mean path length, and mean velocity of $\mathrm{X}$ and Y-axis (Table 2). All parameters except for area surrounding mean path length were calculated by step number because of the difference of stepping frequency among the various conditions.

\subsubsection{Stepping Parameters}

The time difference between fixed tempo and stepping was selected as the evaluation parameter for dynamic balance [16]. Moreover, single and double support times and swing time proposed in previous studies were selected as the parameters to reflect the stepping strategy $[15,18]$. Figure 1 shows the characteristics of the stepping parameters in this study. Each parameter was calculated by dividing the total time (20 s) by total step number. The mean time difference was calculated by dividing the total time difference by the total step number.

Table 2. COP parameters.

\begin{tabular}{|c|c|}
\hline Parameters & The property of parameters \\
\hline \multicolumn{2}{|l|}{ Distance } \\
\hline Root mean square of $\mathrm{X}$-axis & Equation: $\sqrt{\frac{\left(\sum \mathrm{X}_{\mathrm{i}}-\overline{\mathrm{X}}\right)^{2}}{\mathrm{~N}}}$ \\
\hline Root mean square of Y-axis & Equation: $\sqrt{\frac{\left(\Sigma Y_{i}-\bar{Y}\right)^{2}}{N}}$ \\
\hline \multicolumn{2}{|l|}{ Area } \\
\hline Area surrounding mean path length & $\begin{array}{l}\text { Area surrounding fringe path } \\
\text { dividing to total path length }\end{array}$ \\
\hline \multicolumn{2}{|l|}{ Velocity } \\
\hline Mean velocity of $\mathrm{X}$-axis sway & $\begin{array}{c}\text { The mean velocity of } \mathrm{X} \text {-, } \mathrm{Y} \text {-axis } \\
\text { for COP sway }\end{array}$ \\
\hline & \\
\hline
\end{tabular}

All parameters were calculated the values per step. 


\subsubsection{Data Analysis}

To examine the trial-to-trial reliability of each stepping parameter, the single measure intra-class correlation coefficient (ICC) by one way ANOVA was calculated. Repeated one-way ANOVA was used to reveal the differences among tempos. Moreover, if there was a significant difference among tempos, multiple comparisons were examined by Tukey's HSD method. Pearson's correlation coefficient was calculated among stepping and COP parameters. A probability level of 0.05 was indicative of statistical significance.

\section{RESULTS}

The ICCs of stepping and COP parameters were high (ICC $=0.75-0.97$ ).

Figure 2 shows the typical COP sway in each tempo. Although COP sways at 60 and $120 \mathrm{bpm}$ tempos moved regularly and periodically according to right and left steps, those at $40 \mathrm{bpm}$ tended to cause unexpected COP sway during single leg support phase and to produce variation in transfer trajectory of center of gravity.

Table 3 shows the result of repeated one-way ANOVA for stepping and COP parameters among tempos. There

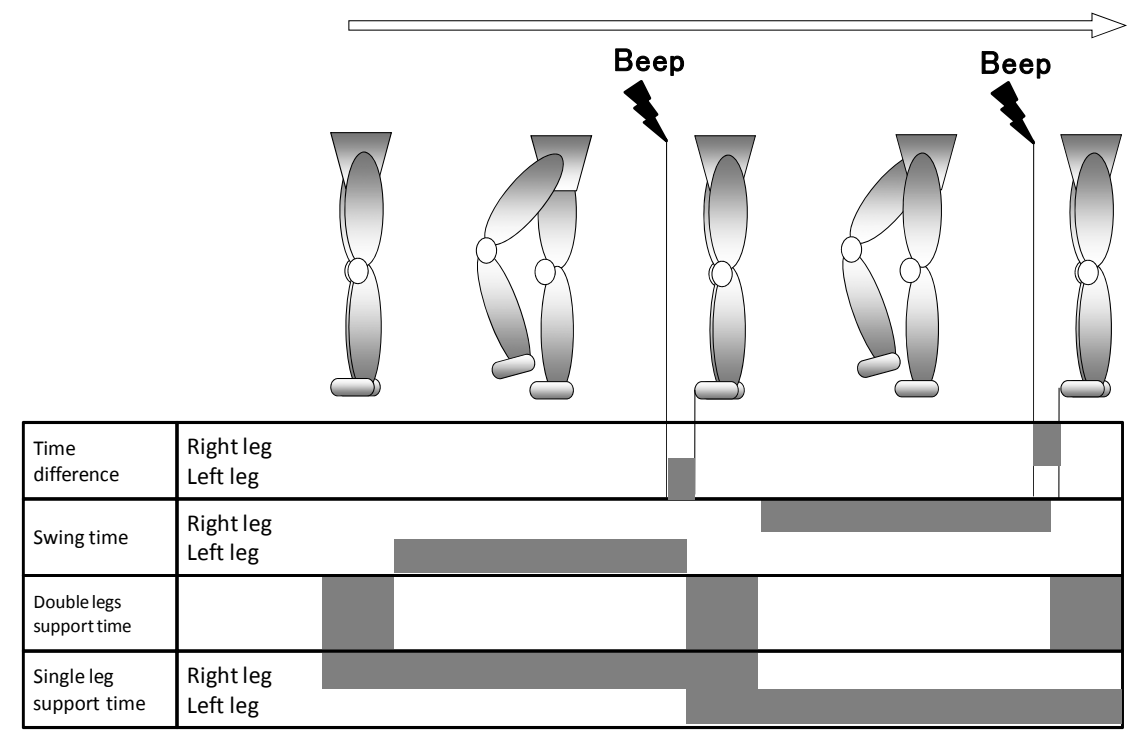

Figure 1. Characteristics of stepping parameters. Each parameter was calculated by dividing the amount time by total step number.
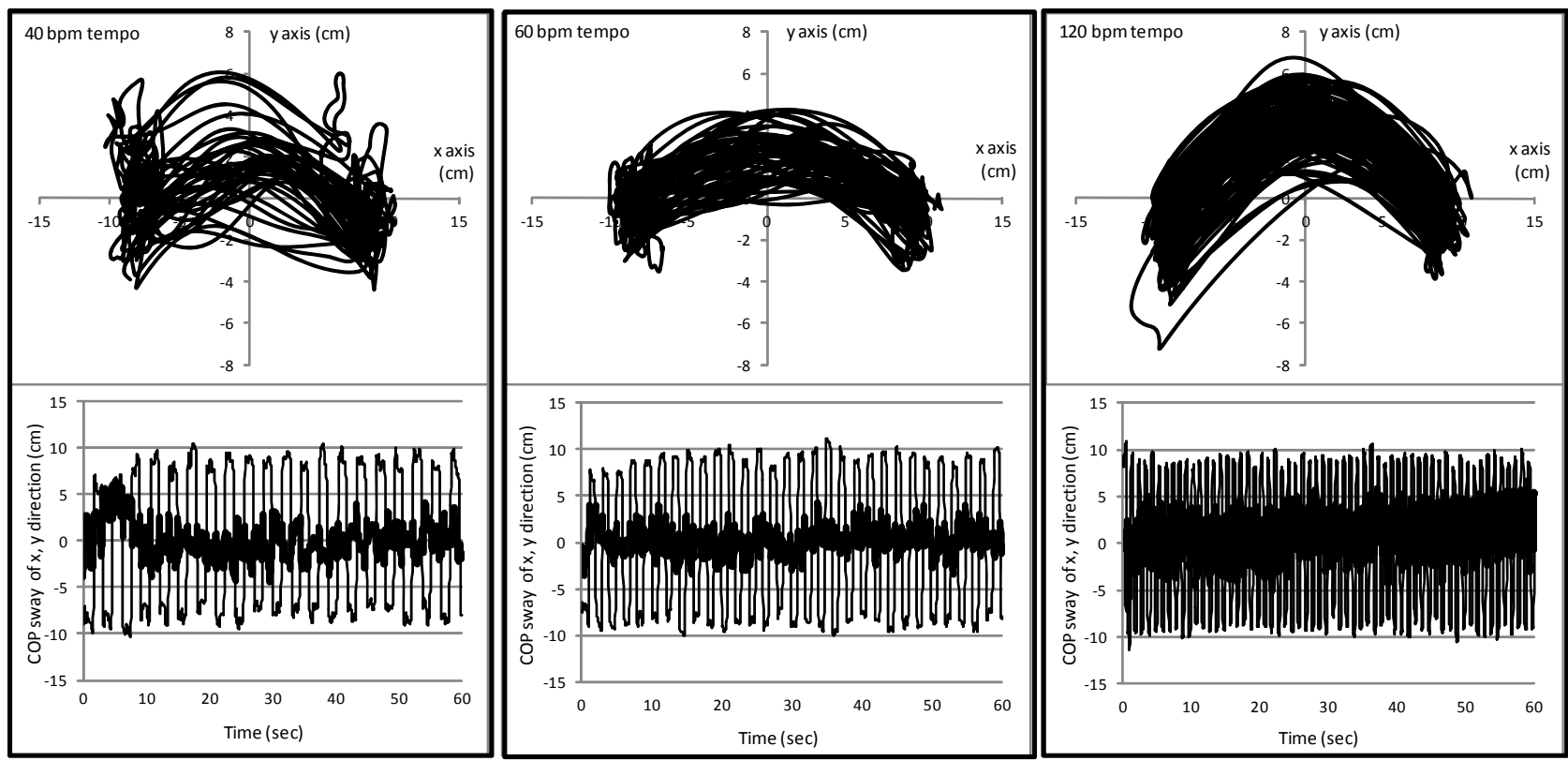

Figure 2. Typical COP sway in each stepping tempo. Upper panels: COP sway in two dimentional coordinate scaling for each stepping tempo. Lower panels: COP sway by $\mathrm{x}$, y direction (thin line: $\mathrm{x}$ direction, heavy line: $\mathrm{y}$ direction) for each stepping tempo. 
were significant differences in all parameters except for the root mean square of the $\mathrm{X}$-axis. All stepping parameters became significantly larger at slower tempos. Of the COP parameters, velocity parameters (X and Y-axis) became larger at slower tempos, but the root mean square of the Y-axis and the area surrounding mean path length decreased.

Table 4 shows the correlations among stepping and COP parameters in each tempo. There was no significant correlation at $40 \mathrm{bpm}$. At 60 and $120 \mathrm{bpm}$, the root mean square and the velocity of the $\mathrm{X}$-axis correlated significantly with single support and swing time $(\mathrm{r}=|0.45-0.53|)$.

\section{DISCUSSION}

One of the main causes of falls in the elderly is loss of balance caused by missteps and slips during walking [3]. Hence, movement tasks that involve transferring body gravity and supporting the body with one leg are useful for evaluating the elderly's dynamic balance. Shin and Demura [16] proposed a stepping task adapted to a fixed tempo to evaluate dynamic balance of the elderly. A step-

Table 3. Repeated one-way ANOVA for stepping and COP parameters among stepping tempos.

\begin{tabular}{|c|c|c|c|c|c|c|c|c|c|c|c|c|c|}
\hline \multirow[b]{2}{*}{ Parameters } & & \multicolumn{3}{|c|}{$40 \mathrm{dpm}$} & \multicolumn{3}{|c|}{$60 \mathrm{dpm}$} & \multicolumn{3}{|c|}{$120 \mathrm{dpm}$} & \multirow[b]{2}{*}{ F-value } & \multirow[b]{2}{*}{$\mathrm{p}$} & \multirow[b]{2}{*}{ HSD } \\
\hline & & ICC & mean & SD & ICC & mean & SD & ICC & mean & $\mathrm{SD}$ & & & \\
\hline \multicolumn{14}{|l|}{ Stepping parameters } \\
\hline $\begin{array}{l}\text { Time difference between fixed } \\
\text { tempo and stepping }\end{array}$ & (s) & 0.75 & 0.06 & 0.01 & 0.78 & 0.04 & 0.01 & 0.76 & 0.02 & 0.00 & 115.95 & $0.00^{*}$ & $120<60<40$ \\
\hline Single leg support times & (s) & 0.85 & 1.70 & 0.06 & 0.93 & 1.19 & 0.05 & 0.93 & 0.65 & 0.02 & 5835.73 & $0.00^{*}$ & $120<60<40$ \\
\hline Double legs support times & (s) & 0.85 & 0.27 & 0.06 & 0.86 & 0.22 & 0.05 & 0.94 & 0.16 & 0.03 & 60.37 & $0.00^{*}$ & $120<60<40$ \\
\hline \multicolumn{14}{|l|}{ COP parameters } \\
\hline Root mean square of $\mathrm{X}$-axis & $(\mathrm{cm})$ & 0.89 & 0.11 & 0.01 & 0.95 & 0.11 & 0.01 & 0.94 & 0.11 & 0.01 & 2.29 & 0.12 & \\
\hline Root mean square of Y-axis & $(\mathrm{cm})$ & 0.79 & 0.03 & 0.01 & 0.83 & 0.03 & 0.01 & 0.85 & 0.04 & 0.01 & 34.60 & $0.00^{*}$ & $40,60<120$ \\
\hline $\begin{array}{l}\text { Area surrounding mean path } \\
\text { length }\end{array}$ & $(1 / \mathrm{cm})$ & 0.80 & 0.15 & 0.03 & 0.89 & 0.19 & 0.04 & 0.89 & 0.29 & 0.07 & 120.43 & $0.00^{*}$ & $40<60<120$ \\
\hline Mean velocity of $X$-axis sway & $(\mathrm{cm} / \mathrm{s})$ & 0.88 & 0.29 & 0.03 & 0.97 & 0.27 & 0.03 & 0.96 & 0.25 & 0.02 & 46.46 & $0.00^{*}$ & $120<60<40$ \\
\hline Mean velocity of Y-axis sway & $(\mathrm{cm} / \mathrm{s})$ & 0.88 & 0.18 & 0.03 & 0.95 & 0.16 & 0.03 & 0.92 & 0.16 & 0.04 & 11.65 & $0.00^{*}$ & $60,120<40$ \\
\hline
\end{tabular}

*: Statistical significant level were adjusted by Bonferroni method; ICC: Intracrass correlation coefficient.

Table 4. Correlations among stepping and COP parameters in each tempo.

\begin{tabular}{|c|c|c|c|c|c|c|c|}
\hline \multirow[b]{3}{*}{ Parameters } & \multicolumn{3}{|c|}{ Root mean square } & \multirow{2}{*}{$\begin{array}{c}\text { Area } \\
\text { Mean path length }\end{array}$} & \multicolumn{3}{|c|}{ Mean velocity } \\
\hline & \multicolumn{2}{|l|}{$\mathrm{X}$-axis } & \multirow[t]{2}{*}{ Y-axis } & & \multicolumn{2}{|l|}{$\mathrm{X}$-axis } & \multirow[t]{2}{*}{ Y-axis } \\
\hline & & & & & & & \\
\hline \multicolumn{8}{|l|}{$40 \mathrm{bpm}$} \\
\hline Time difference between fixed tempo and stepping & 0.17 & & -0.33 & 0.02 & 0.02 & & -0.03 \\
\hline Single leg support times & 0.06 & & -0.13 & 0.09 & 0.15 & & 0.29 \\
\hline Swing time & -0.09 & & 0.10 & -0.07 & -0.18 & & -0.30 \\
\hline Double legs support times & 0.03 & & -0.13 & 0.09 & 0.13 & & 0.21 \\
\hline \multicolumn{8}{|l|}{$60 \mathrm{bpm}$} \\
\hline Time difference between fixed tempo and stepping & 0.35 & & -0.23 & 0.20 & 0.25 & & -0.05 \\
\hline Single leg support times & 0.45 & * & 0.12 & -0.12 & 0.46 & $*$ & 0.12 \\
\hline Swing time & -0.45 & $*$ & -0.10 & 0.11 & -0.47 & $*$ & -0.07 \\
\hline \multicolumn{8}{|l|}{120 bpm } \\
\hline Time difference between fixed tempo and stepping & 0.31 & & -0.09 & 0.08 & 0.33 & & -0.11 \\
\hline Single leg support times & 0.53 & $*$ & 0.27 & -0.27 & 0.53 & $*$ & 0.21 \\
\hline Swing time & -0.48 & * & -0.25 & 0.24 & -0.47 & $*$ & -0.21 \\
\hline Double legs support times & 0.42 & & 0.25 & -0.23 & 0.42 & & 0.22 \\
\hline
\end{tabular}


ping test involving accurate adaptation to a fixed tempo is very easy for young people but not for the elderly with inferior lower body strength and dynamic balance. This study examined trial-to-trial reliability of step tests using three fixed tempos reported by Shin and Demura [16] for young adults. All parameters showed high reliability at all tempos.

A stepping motion is performed periodically and is divided into the leg support and swing phases [19]. The former is further divided into both support phases by one leg and both legs. The single support time reflects the leg support phase and shows the time until shift of the body's center of gravity to another leg. In addition, stepping movements are performed based on the subject's step strategy in which they make the most stable step motions. Although their stepping strategy may differ among tempos, the reproducibility of the stepping parameters selected in this study is high. Shin and Demura [16] suggested that the difficulty of a stepping task involving adaptation to a fixed tempo depends on tempo velocity. Since the stepping task used in this study was easy for young adults, the reliability among tempos may have not differed.

Maki [20] reported that the following gait changes appear with age: a decrease of walk velocity, stride, cadence, and the center of gravity moving up and down; an increase of both legs connecting time; and decreased coordination of the upper and lower limbs. Namely, the steps of the elderly are neither rapid and strong nor smooth and coordinated. Unlike mobility tests in which the elderly can walk or step with preferred or maximal velocity, the present stepping test controlled by a fixed tempo would further disturb smooth and coordinated stepping movements. Because the $120 \mathrm{bpm}$ tempo, the most rapid tempo, produces larger body sway, it was assumed to be the most difficult. The present result showed that the COP path length in an anteroposterior direction was the largest at $120 \mathrm{bpm}$. In short, the above stated hypothesis was supported. However, sway area and velocity parameters were smaller at $120 \mathrm{bpm}$ than at 40 bpm, and the time difference between foot contact and fixed tempo was larger with slower tempo. Thus, the difficulty of the stepping task may be the largest at the 40 bpm tempo. Toyama and Fujiwara [17] reported that the fastest tempo of $120 \mathrm{bpm}$ selected in this study is close to a walking tempo and can be performed with highly stable stepping. Shin and Demura [16] clarified that the 120 bpm tempo is the best for stable stepping in both the elderly and the young. At the 60 and $120 \mathrm{bpm}$ tempos, single support and swing times reflecting the stepping strategy correlated significantly with COP path distance and velocity in an anteroposterior direction $(\mathrm{r}=|0.45-0.53|)$. Because these tempos are affected largely by the automation of stepping, single support and swing phases ap- peared at a constant frequency. In short, it is inferred that these parameters related with COP parameters because the stepping strategy was stable during stepping.

Moreover, the stepping tempo at $40 \mathrm{bpm}$ showed the largest sway velocity in an anteroposterior and horizontal direction and the smallest surrounding area mean path length $\left(\mathrm{cm} / \mathrm{cm}^{2}\right.$ : total path length/surrounding area). This suggests that stepping at $40 \mathrm{bpm}$ induced a larger sway area in relation to total path length and a faster COP sway. In other words, the stepping tempo at $40 \mathrm{bpm}$ may induce unexpected body sway. Because stepping at $40 \mathrm{bpm}$ requires a long single leg supporting time and maintains an unstable body posture, accidental body sway appears and exertion of dynamic balance ability is required. Hence, the difficulty of the stepping task may be higher at the 40 bpm tempo than at the 60 and $120 \mathrm{bpm}$ tempos.

In conclusion, COP sway during stepping as well as the time difference between foot contact time and fixed tempo increase at slower tempos. The center of body gravity while stepping at $40 \mathrm{bpm}$ tends to be larger with horizontal direction, and this tempo may be useful for the evaluation of dynamic balance ability.

\section{REFERENCES}

[1] Oliver, D. (2007) Older people who fall: Why they matter and what you can do. British Journal of Community Nursing, 12, 500-507.

[2] Kikuchi, R., Kozaki, K., Nakamura, T. and Toba, K. (2008) Muscle and bone health as a risk factor of fall among the elderly. An approach to identify high-risk fallers by risk assessment. Clinical Calcium, 18, 784-787.

[3] Cutson, T.M. (1994) Falls in the elderly. American Family Physicians, 49, 149-156.

[4] Banez, C., Tully, S., Amaral, L., Kwan, D., Kung, A., Mak, K., Moghabghab, R. and Alibhai, S.M. (2008) Development, implementation, and evaluation of an Interprofessional Falls Prevention Program for older adults. Journal of the American Geriatrics Society, 56, 15491555. doi:10.1111/j.1532-5415.2008.01790.x

[5] van der Velde, N., Stricker, B.H., Pols, H.A. and van der Cammen, T.J. (2007) Withdrawal of fall-risk-increasing drugs in older persons: Effect on mobility test outcomes. Drugs and aging, 24, 691-699. doi:10.2165/00002512-200724080-00006

[6] Gill, J., Allum, J.H., Carpenter, M.G., Held-Ziolkowska, M., Adkin, A.L., Honegger, F. and Pierchala, K. (2001) Trunk sway measures of postural stability during clinical balance tests: Effects of age. The Journals of Gerontology. Series A, Biological Sciences and Medical Sciences, 56, M438-M447. doi:10.1093/gerona/56.7.M438

[7] Graham, R.C., Smith, N.M. and White, C.M. (2005) The reliability and validity of the physiological cost index in healthy subjects while walking on 2 different tracks. Archives of Physical Medicine and Rehabilitation, 86, 20412046. doi:10.1016/j.apmr.2005.04.022 
[8] Newton, R.A. (1997) Balance screening of an inner city older adult population. Archives of Physical Medicine and Rehabilitation, 78, 587-591. doi:10.1016/S0003-9993(97)90423-8

[9] Rubenstein, L.Z., Josephson, K.R., Trueblood, P.R., Yeung, K., Harker, J.O. and Robbins, A.S. (1997) The reliability and validity of an obstacle course as a measure of gait and balance in older adults. Aging (Milano), 9, 127135.

[10] Medell, J.L. and Alexander, N.B. (2000) A clinical meassure of maximal and rapid stepping in older women. The Journals of Gerontology. Series A, Biological Sciences and Medical Sciences, 55, M429-M433. doi:10.1093/gerona/55.8.M429

[11] Cho, B.L., Scarpace, D. and Alexander, N.B. (2004) Tests of stepping as indicators of mobility, balance, and fall risk in balance-impaired older adults. Journal of the American Geriatrics Society, 52, 1168-1173. doi:10.1111/j.1532-5415.2004.52317.x

[12] Demura, S., Yamaji, S. and Kitabayashi, T. (2005) Gender and age-related differences of dynamic balancing ability based on various stepping motions in the healthy elderly. Journal of Human Ergology (Tokyo), 34, 1-11.

[13] Nnodim, J.O., Strasburg, D., Nabozny, M., Nyquist, L., Galecki, A., Chen, S. and Alexander, N.B. (2006) Dynamic balance and stepping versus tai chi training to improve balance and stepping in at-risk older adults. Journal of the American Geriatrics Society, 54, 1825-1831. doi:10.1111/j.1532-5415.2006.00971.x
[14] Maki, B.E., Perry, S.D., Scovil, C.Y., Peters, A.L., McKay, S.M., Lee, T.A., Corbeil, P., Fernie, G.R. and McIlroy, W.E. (2008) Interventions to promote more effective balance-recovery reactions in industrial settings: New perspectives on footwear and handrails. Industrial Health, 46, 40-50. doi:10.2486/indhealth.46.40

[15] Demura, S., Yamada, T. and Shin, S. (2008) Age and sex differences in various stepping movements of the elderly. Geriatrics \& Gerontology International, 8, 180-187. doi:10.1111/j.1447-0594.2008.00468.x

[16] Shin, S. and Demura, S. (2007) Effective tempo of the step test for dynamic balance ability in the elderly. Journal of Physiological Anthropology, 26, 563-567. doi:10.2114/jpa2.26.563

[17] Toyama, H. and Fujiwara, K. (1990) Interference of upper limbs exercise with different automatized levels. Physical Fitness Sports and Medicine, 39, 44-52.

[18] Murray, M.P., Kory, R.C. and Clarkson, B.H. (1969) Walking patterns in healthy old men. Journal of Gerontology, 24, 169-178.

[19] Commissaris, D.A., Nieuwenhuijzen, P.H., Overeem, S. de Vos, A., Duysens, J.E. and Bloem, B.R. (2002) Dynamic posturography using a new movable multidirectional platform driven by gravity. Journal of Neuroscience Methods, 113, 73-84. doi:10.1016/S0165-0270(01)00477-0

[20] Maki, B.E. (1997) Gait changes in older adults: Predictors of falls or indicators of fear. Journal of the American Geriatrics Society, 45, 313-320. 